\title{
Investigating Atmospheric Sulfuric Acid-Water-Ammonia Particle Formation Using Quantum Chemistry
}

\section{Theo Kurtén $^{*}$ and Hanna Vehkamäki *}

$\begin{array}{lr}\text { Contents } & 407 \\ \text { 1. Introduction } & 411 \\ \text { 2. Theoretical Methods for Free Energy Calculations } & 415 \\ \text { 3. Applications of Quantum Chemistry to Atmospheric Nucleation } & \\ & \text { Phenomena } \\ 3.1 \text { Construction of classical potentials } & 415 \\ 3.2 \text { Investigating the fundamental chemistry of nucleating systems } & 416 \\ 3.3 \text { Computing formation free energies for nucleating clusters } & 422 \\ \text { 4. Challenges } & 423 \\ \text { 5. Conclusions } & 424 \\ \text { Acknowledgements } & 425 \\ \text { References } & 425\end{array}$

\section{INTRODUCTION}

The scope of this paper is to provide an overview of methods used to study properties of electrically neutral molecular clusters initiating particle formation in the troposphere, with focus on quantum chemistry. The review of results is intended to be complete with regard to water-sulfuric acid-ammonia clusters. Concerning studies on clusters including other molecular species, we review representative examples and newest publications. Ionic clusters and clusters involving iodine, related to coastal nucleation, are mentioned in passing.

Atmospheric aerosols are liquid or solid particles floating in the air. Their size ranges from nanometer-scale molecular clusters to, for example, road dust with a

* Department of Physical Sciences, P.O. Box 64, 00014 University of Helsinki, Finland

E-mail: theo.kurten@helsinki.fi

E-mail: hanna.vehkamaki@helsinki.fi 
diameter of a few hundred micrometers. Cloud droplets are also aerosols with diameters above one micrometer, and they always contain a non-aqueous aerosol particle as a condensation nucleus. Aerosol particles can be emitted to the atmosphere by both natural and anthropogenic processes, for example desert dust and soot from combustion, respectively. Gaseous condensable vapors can condense, or heterogeneously nucleate, on the surfaces of existing particles, changing their size and compositions, but vapors can also form completely new particles in the atmosphere by homogeneous nucleation. The smallest ultrafine atmospheric particles are products of gas-to-particle nucleation, and over continents it has been estimated that $30 \%$ of the aerosol particles are formed this way [1].

Aerosols affect climate, visibility and human health. The fourth assessment report of the Intergovernmental Panel on Climate Change [2] concludes that anthropogenic contributions to aerosols together produce a cooling effect, and aerosols remain the dominant uncertainty in predicting radiative forcing. Aerosols affect our planets radiation budget by scattering and absorbing incoming solar radiation as well as outgoing long-wave radiation. Since cloud formation depends on the aerosol present, they also have an indirect effect on climate by influencing the occurrence, properties and lifetime of clouds and precipitation.

Although the ultrafine aerosol particles constitute a negligible fraction of the total aerosol mass, they make up a significant fraction of the number concentration. In polluted urban conditions, traffic-related nucleated particles can even dominate the aerosol number distribution. The smaller the particles, the deeper they penetrate into the respiratory passages, and there is evidence that nano-scale particles can find their way from the lungs to the circulations system, causing cardiovascular diseases and premature deaths [3]. Despite the evidence of the importance of high concentrations of ultrafine particles in aerosol related phenomena, present legislative constraints do not control the number concentration, only the mass of aerosol particles. In most countries, current legislation restricts the total mass of particles below 10 micrometers in aerodynamic diameter (PM10); legal limitations on the total mass of particles below 2.5 micrometer in diameter (PM2.5) are in effect only in the United States of America. WHO has guidelines for the upper limit of PM2.5, and binding restrictions on PM2.5 are currently in preparation in the European Union. Before limitations on the number concentrations can be prepared, scientist need to achieve a significantly improved understanding of the role of both natural and anthropogenic ultrafine particles, and their measurements techniques; the road to this objective starts from understanding the formation processes and properties of atmospheric ultrafine particles.

Particle formation events from gaseous precursors are observed frequently almost everywhere in the troposphere, both in polluted cities and remote clean areas [4]. It is likely that different nucleation mechanisms are at work in different conditions, but no formation mechanism has been identified so far. It is, however, clear that particles are formed by nucleation of a multicomponent vapor mixture. Water vapor is the most abundant condensable gas in the atmosphere, but it can not form particles on its own: homogeneous nucleation requires such a high supersaturation, that heterogeneous nucleation on omnipresent pre-existing particles always starts first and consumes the vapor. However, vapor that is un- 
dersaturated with respect to pure liquids, for example sulfuric acid and water, can be highly supersaturated with respect to the mixture of these liquids. In coastal areas [5] iodine-containing species are believed to be responsible for particle formation, and in several cases the gas-phase concentration of sulfuric acid correlates well with the appearance of ultrafine particles. In many cases photochemistry and/or mixing induced by sunrise seems to trigger particle formation, although at some sites also night-time nucleation has been observed. Nucleation around atmospheric ions is one promising pathway to explain at least some of the observed formation events [6-8]. Newly nucleated particles contain less than one hundred molecules, possibly only around ten or less. Electrically neutral particles can not even be detected before they have grow to a diameter above $2.5 \mathrm{~nm}$, when they already contain thousands of molecules. The composition of the particles can be uncovered by mass spectrometry only when they contain around one million molecules. At this stage, the substances added during the growth process completely overshadow the composition of the original critical cluster produced by nucleation. Theoretical tools are thus vital for understanding the first stages of particle formation.

The nucleation process is often thought to be simple cluster kinetics: clusters grow when they collide with vapor molecules (monomers) and each other, and break up by evaporating vapor molecules or splitting into two smaller clusters. However, for some organic nucleation mechanisms it is possible that the kinetics involved is significantly more complicated (see below). In most cases it is enough to take into account only monomer-cluster collisions and the evaporation of single molecules. Collisions with inert air molecules keep the clusters close to the ambient temperature despite the latent heat involved in condensation and evaporation of molecules. The critical cluster is the smallest cluster for which growth is more likely than decay. Growth probabilities can for many cluster types be justifiably calculated using simple kinetic gas theory, and the evaporation probability of a cluster depends on the internal stability of the cluster, which is characterized by its formation free energy. The formation free energy is the difference between the free energy of the cluster and the free energies the constituent molecules would have when unbound in the vapor phase, and it depends on the vapor densities as well as the temperature. The formation free energy can be split into an attractive volume term (corresponding to the attractive interactions between cluster molecules) and a repulsive surface term (corresponding to the work required to form the surface of the cluster). Since the volume term depends on the third power and the surface term on the second power of the number of molecules in the cluster, the free energy curve plotted against the number of molecules will exhibit a maximum. The location and height of the maximum depends on the temperature and the concentration of the nucleating vapor. From a macroscopic thermodynamic point of view, the formation free energy can be split into enthalpy and entropy contributions. It should be noted that even though the enthalpy term may, for many substances, always be favourable for cluster formation, it is overweighed by the unfavourable entropy term for small cluster sizes.

When the formation free energy is plotted against the number of molecules, in a one-component vapor the critical cluster size is the location of the maximum of 


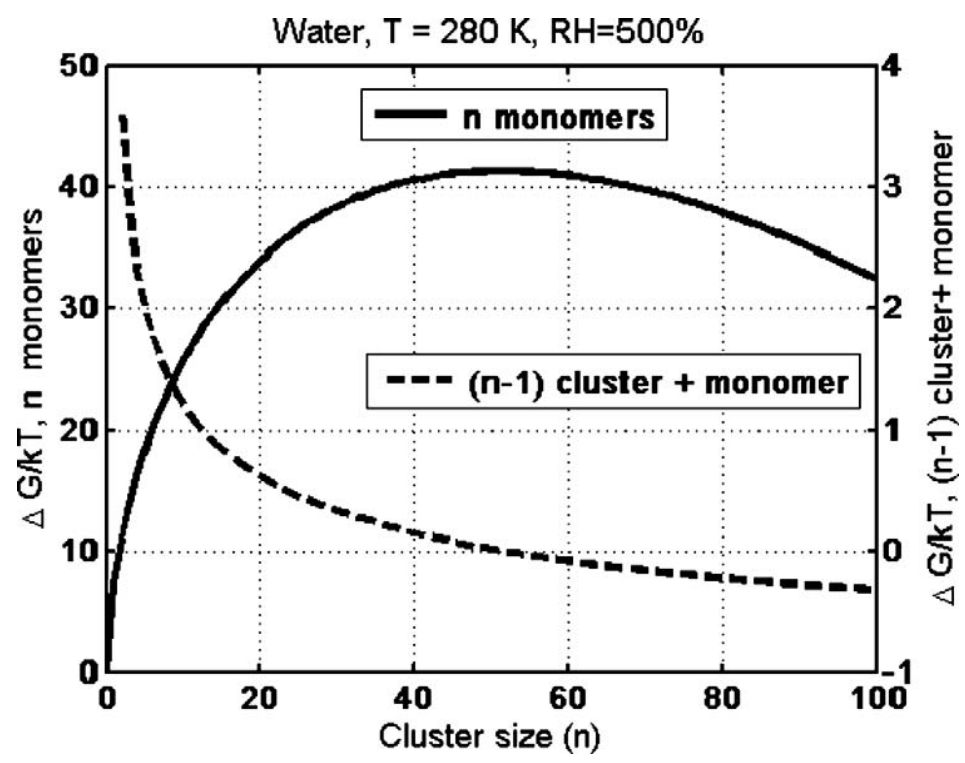

FIGURE 19.1 Free energy surface for cluster formation in the one-component case, here for pure water (see McDonald [9] for original data). Both free energy related to the formation of a cluster from single molecules (monomers) and the free energy of adding one monomer to a cluster are plotted against the number of molecules in the cluster. The free energy of monomer addition is positive for small clusters, but turns negative at the critical size.

the free energy curve. See Figure 19.1 where the free energy curve is plotted using water as an example (see Abraham [10] and Kashchiev [11] for details). In multicomponent systems, one axis per component is needed to represent the number of molecules, and the critical cluster size is the saddle point of the free energy surface: it is a maximum on one direction, but minimum in all other directions. When the cluster has overcome the critical size, it can freely grow by going downhill along the free energy surface in one direction.

It should be noted that the thermodynamic free energy surface seen in Figure 19.1 is somewhat different from the potential energy surfaces commonly encountered in physical chemistry or molecular physics. The latter are plotted with respect to the positions of atomic nuclei (usually expressed in terms of one or more reaction coordinates; see Figure 19.2 for a schematic example). Equilibrium structures are represented as minima on this surface, and the differences between the minima are the thermodynamic barriers. The free energy surfaces encountered in nucleation studies (such as Figure 19.1) can be viewed as a small subset of these potential energy surfaces, where the arrangement of atoms in the clusters is not accounted for explicitly, but is instead implicitly included via the method by which the free energies are calculated. When nucleation rates are calculated from free energy surfaces such as that given in Figure 19.1, it is implicitly assumed that there are no reaction barriers (also called kinetic barriers) for cluster formation processes. (On the atomic potential energy surface, reaction barriers are repre- 


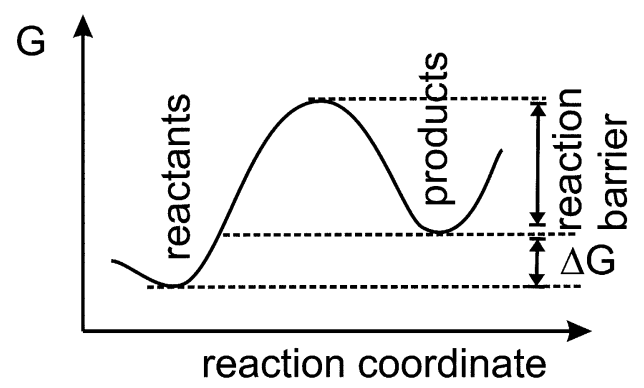

FIGURE 19.2 Schematic free energy surface for a chemical reaction leading from "reactants" to "products". The formation free energies of Figure 19.1 are related to the differences between the minima. (Note that free energies are rigorously defined only for stationary points on the atomic potential energy surface, but are used here loosely to facilitate comparison with the formation free energies of Figure 19.1.)

sented by saddle points, or maxima in the one-dimensional case, for example in Figure 19.2.) As long as the studied systems are weakly bound molecular clusters, this assumption is not problematic. However, as the focus of studies shifts toward cluster formation involving more chemically complex species and bondbreaking reactions, the distinction between thermodynamics (related to minima on the atomic potential energy surface) and kinetics (related to saddle points on the atomic potential energy surface) should be emphasized.

The nucleation rate (number of particles formed per volume and time unit) is inversely proportional to the exponential of the critical cluster formation free energy. Steady-state conditions with time-independent concentrations of vapor and clusters are most often assumed to calculate the nucleation rate, but in reality the process in the atmosphere is highly dynamic: cluster formation consumes the vapor, sources and / or gas-phase chemistry produce it, while condensation on preexisting particles and chemistry can act as vapor sinks. Fully dynamic studies of actual cluster formation processes in atmospheric varying conditions are desirable in the future, if or when computer resources permit them, but presently theoretical efforts are focused on predicting the formation free energy of the critical cluster. Besides the interaction energy of the molecules, also the entropic contributions arising from vibrations and rotations at atmospheric temperature far above $0 \mathrm{~K}$ must be determined. The size- and composition-dependent properties of real atmospheric clusters are averages over countless cluster configurations populated at ambient conditions, not properties of the most stable minimum free energy structures.

\section{THEORETICAL METHODS FOR FREE ENERGY CALCULATIONS}

The most widely used method for calculating the formation free energy is the classical nucleation theory (CNT) [12-16] based on thermodynamics. The molecular clusters are treated as spherical droplets of bulk liquid having sharp boundaries 
associated with surface tension. The dominant role of CNT is understandable since it is applicable as soon as a few key properties of the liquid forming the droplet are known: liquid density, saturation vapor pressures of all the components above the mixture, and the surface tension. It should be noted that even this basic information is not available for many of the atmospherically interesting molecular mixtures, for example highly concentrated aqueous solutions of ammonia and sulfuric acid, let alone mixtures of, for example, water, sulfuric acid and various organic molecules. The fact that bulk liquid density is used to model a small molecular cluster can be corrected for within CNT, but the use of size-independent flat surface tension causes inaccuracies and, in the case of surface active mixtures where certain components accumulate in the surface layer, even a complete breakdown of the theory. For mixtures that are not surface active, CNT is valid down to surprisingly small clusters, for example 8-10 molecules in the case of pure water [17].

Classical density functional theory (DFT) $[18,19]$ treats the cluster formation free energy as a functional of the average density distributions of atoms or molecules. The required input information is an intermolecular potential describing the substances at hand. The boundary between the cluster and the surrounding vapor is not anymore considered sharp, and surface active systems can be studied adequately. DFT discussed here is not to be confused with the quantum mechanical density functional theory (discussed below), where the equivalent of the Schrödinger equation is expressed in terms of the electron density. Classical DFT has been used successfully to uncover why and how CNT fails for surface active systems using simple model molecules [20], but it is not practically applicable to real atmospheric clusters: if the molecules are not chain-like, the numerical solution of the problem gets too burdensome, unless the whole molecule is treated in terms of an effective potential.

Molecular dynamics (MD), also knows as molecular mechanics (MM), simulations [21] solve Newton's equations of motion for the chosen set of molecules, and Monte Carlo (MC) simulations [22-24] study statistical averages of cluster properties at certain conditions, for example, constant vapor concentration and temperature. Both single clusters, clusters surrounded by vapor phase, and whole cluster distributions immersed into vapor can be studied. The larger the number of molecules, the higher the computational cost. The input information required is again the potential energy related to molecular interactions, including both intermolecular and intramolecular contributions in the case of non-rigid molecules. In principle these interactions can be calculated using quantum chemistry, but enormous computational costs of such a method force us mostly to resort to classical force fields to describe the interactions. MD simulations capture the true dynamic nature of cluster formation. MD can answer questions about timescales of various processes, for example, the rate of evaporations/condensation, relaxation time or the lifetime of a cluster [25]. A truly dynamic method is the only way to calculate the actual nucleation rate, the time required to form a critical cluster when starting with supersaturated vapor. The problems of MD are related to moderately high computational costs (compared to CNT or MC, though not to quantum chemical methods), incomplete sampling of cluster configurations, and temper- 
ature control. Established thermostatting methods may not work well for small clusters [26,27], and the most realistic way of temperature control with an excess of inert gas molecules requires a lot of computing time. MC methods used in atmospheric nucleation studies are computationally less expensive, and can thus be applied to larger systems, and/or to gather more statistics. Temperature control is trivial, but we lose information on the realistic temperature fluctuations in the growing clusters. MC methods are well suited to calculations of average formation free energies and critical cluster sizes, but they yield no information about the absolute timescales of the cluster formation process. $\mathrm{MC}$ is an effective tool for sampling the configuration space.

Classical interaction potentials for molecular systems can be pairwise or take manybody interactions into account, molecules can be rigid or flexible, and both stretching and torsion motions can be considered [28]. Polarizability can be introduced, but for example for water this does not seem to make the potentials mimic reality unambiguously better. The potentials, also called force fields, are fitted to reproduce certain sets of properties, and can fail to reproduce other characteristics. The potentials suffer from problems with transferability: for example a water-sulfuric acid potential developed for the two-component mixture does not necessarily describe the interaction between water and sulfuric acid in a threecomponent mixture of water, sulfuric acid and ammonia. Also, potentials created for bulk liquid do not always work for surface layers or small clusters.

Quantum chemistry, also called electronic structure methods, represents the most chemically accurate, but also computationally expensive, simulation method. Whereas classical potentials treat electronic interactions only implicitly, quantum chemical methods are based on the numerical solution of the Schrödinger equation (or the related Kohn-Sham equations in case of DFT) for systems of nuclei and electrons, subject to a number of approximations with varying degrees of severity. For all methods applicable to the (relatively large) molecular clusters of interest to atmospheric nucleation studies, these approximations include, for example, treating the motion of atomic nuclei using classical mechanics (with the forces computed from the electronic wavefunction), and expressing the electronic wavefunction in terms of single-electron wavefunctions composed of basis functions selected from some basis set. In studies of atmospheric molecular clusters, this basis set usually consists of atom-centered gaussian functions, though slater-type functions, numerical basis functions and plane waves have also been used. Electron-electron correlation has to be accounted for if chemical accuracy is desired. This is done either through an exchange-correlation functional (in quantum density functional theory) or via the use of various excitation operators (in correlated $a b$ initio methods). Due to their prohibitively high computational costs, only the lowest-order correlated $a b$ initio methods such as MP2 (2nd order Møller-Plesset perturbation theory) can generally be applied to systems of atmospheric interest, though single-point energy corrections can sometimes be computed at higher levels such as MP4 or CCSD(T) (see e.g. Jensen [29] for definitions and explanations of the various methods). Hartee-Fock calculations, which ignore electronic correlation, have also been performed for some atmospherically relevant molecular clusters, but as their computational cost is roughly similar to 
DFT methods while their accuracy is significantly lower, they are seldom used anymore.

The advantages and disadvantages of density functional theory in describing hydrogen-bonded and other weakly bound systems has been extensively debated (see e.g. Refs. [30,31]), but there is no clear consensus as to whether or not DFT methods are capable of describing hydrogen bonds as well as for example MP2. For some sets of reference molecules, heavily parametrized density functionals (see e.g. Ref. [32]) often replicate experimental binding energies and/or molecular geometries very well, but analogously to classical potentials, their transferability to different types of systems is questionable. MP2 and related methods may have greater mean errors in comparison to some reference datasets, but they are more systematically reliable. Unlike density functionals, the results can also be systematically improved upon by using higher-order corrections, though these are often computationally too expensive to be of practical use.

The greatest advantage of quantum chemistry in molecular simulations is that there are no system-specific parameters to be determined, and the methods can thus-with the possible exception of the heavily parametrized functionals mentioned above-be applied to any chemical system. In principle, quantum chemistry methods can do everything that classical methods can, but the computational time required for one energy or gradient evaluation is typically several orders of magnitude higher, which usually restricts the dynamical sampling to simple energy minimizations, with thermal contributions to enthalpies and entropies being computed using very simple rigid rotor and harmonic oscillator models. Exceptions to this rule exist, for example Choe et al. [33] very recently used quantum chemistry methods (PBE functional with periodic boundary conditions, pseudopotentials and an adaptive-finite element basis) to study the dynamics of proton transfer reactions of aqueous sulfuric acid solutions. Also, recently developed [34-36] "black box" - applications have made anharmonic vibrational frequency evaluations possible for at least the smallest cluster structures. For example, anharmonic vibrational frequencies have been computed for the $\mathrm{H}_{2} \mathrm{SO}_{4} \cdot \mathrm{H}_{2} \mathrm{O}$ cluster by Miller et al. [37], and for the $\mathrm{H}_{2} \mathrm{SO}_{4} \cdot\left(\mathrm{H}_{2} \mathrm{O}\right)_{2}$ and $\mathrm{HSO}_{4}^{-} \cdot\left(\mathrm{H}_{2} \mathrm{O}\right)_{2}$ clusters by Kurtén et al. [38].

One application for which quantum chemical methods are inherently superior is the study of nucleation processes involving proper chemical reactions (i.e. the breaking and formation of covalent bonds). Classical force fields are usually by construction unable to treat bond breaking and formation, though some potential schemes exist which enable modeling chemical reactions: for covalent molecular systems the most widely used ones are those based on the Brenner and ReaxFF potentials $[39,40]$. While sulfuric acid-water-ammonia cluster formation probably does not involve other reactions than proton transfer (discussed below), it is possible that nucleation mechanisms involving organic molecules or some unstable precursors of sulfuric acid may play significant roles in the atmosphere. These mechanisms can probably not be understood without accounting for complicated chemical reactions that can not be modeled by any classical interaction potentials.

Atmospherically relevant molecular clusters often contain strongly acidic or basic molecules (e.g. sulfuric acid and ammonia), which gives rise to various dif- 
ferent proton transfer reactions. Accurate modeling of proton transfer and its role in cluster thermodynamics and/or kinetics is one of the key challenges for all simulation methods. Classical nucleation theory implicitly assumes that the extent of proton transfer in small cluster is equal to that in bulk liquid. Though classical potentials generally have difficulties in describing bond breaking and formation, there are various tricks that can be used to model proton transfer, as described in Section 3.1. Quantum chemical methods are able to model bond breaking and formation without trouble, and can accurately predict the relative energetics of clusters with different proton transfer states. Dynamical features of proton transfer processes have also been studied using quantum chemical methods [33]. As already mentioned, even quantum chemical methods usually model the motion of nuclei using classical mechanics. Proton transfer reactions are known [41] to involve a large degree of quantum-mechanical tunneling. While tunneling processes can be modeled by applying advanced kinetics models to the computed potential energy surfaces (see e.g. Ref. [42], where this is done for the atmospherically relevant $\mathrm{SO}_{3}+\mathrm{H}_{2} \mathrm{O}$ reaction), these methods are computationally too demanding to be routinely used in simulations of clusters containing multiple molecules. Therefore, though quantum chemical studies of molecular clusters capture the energetics of proton transfer states correctly, it is uncertain whether they correctly describe the dynamics of the process even when dynamical simulations are attempted.

\section{APPLICATIONS OF QUANTUM CHEMISTRY TO ATMOSPHERIC NUCLEATION PHENOMENA}

\subsection{Construction of classical potentials}

One of the most straightforward applications of quantum chemistry to nucleation phenomena is the construction of pair potentials for molecular dynamics studies. Geometry optimizations and binding energies computed using quantum chemical methods can be used to determine all of the potential parameters, as done for example by Ding et al. [43] in their $\mathrm{H}_{2} \mathrm{SO}_{4}-\mathrm{HSO}_{4}^{-}-\mathrm{H}_{2} \mathrm{O}-\mathrm{H}_{3} \mathrm{O}^{+}$potential. Ding et al. fitted the parameters of their intermolecular potential consisting of a Coulomb term and a Lennard-Jones 6-12 term to reproduce the energies and geometries obtained by Re et al. [44]. The intramolecular degrees of freedom were represented by a harmonic potential with the equilibrium distances matching those of Re et al. Another alternative is to determine only part of the parameters, or the relations between the parameters, using quantum chemistry, and fit the rest to experimental results. This was done by Kusaka et al. [45] for the $\mathrm{H}_{2} \mathrm{SO}_{4}-\mathrm{HSO}_{4}^{-}-\mathrm{H}_{2} \mathrm{O}-\mathrm{H}_{3} \mathrm{O}^{+}$system and Kathmann and Hale [46] in their potential for $\mathrm{SO}_{4}^{2 \delta-}-\mathrm{H}_{2} \mathrm{O}-\mathrm{H}^{\delta+}$, where $\delta$ is a partial, empirical charge less than 1 e. Like Ding et al., both groups used Coulomb and Lennard-Jones 6-12 potential terms, but Kusaka et al. used experimental geometries and dipole moments together with the quantum chemical results of Kurdi and Kochanski [47] as a basis for their potential, while Kathmann and Hale based their potential on experimental dipole moments, liquid solution surface tension and partial equilibrium vapor pressures, and their own quantum chemical results (at the $\mathrm{HF} / \mathrm{DZV}+3 \mathrm{P}$ level). 
As mentioned above, the modeling of proton transfer reactions with classical potentials is problematic. There are two main pathways to describe the different protolysis states of sulfuric acid with pair potentials. One is to develop separate potentials for $\mathrm{H}_{2} \mathrm{SO}_{4}$ and $\mathrm{HSO}_{4}^{-}$, as done by Ding et al. [43]. Energetic parameters can then in principle be computed by simulating all the different possible proton transfer states and combining the results using some suitable statistical analysis. For example, for a system with two acid molecules, there are three different proton transfer states if only the first proton transfer reaction is considered, and six if also the second, less favorable, reaction is included. Conceivably, this could also be done "on the fly" by using Monte Carlo sampling at certain intervals to determine whether or not a proton transfer step would be energetically favorable at the given molecular configuration. However, the computational demands of such an approach might well outweigh the benefits, and to our knowledge no such model has been published for any atmospherically relevant molecular cluster system.

Another, in principle more chemically justified, method for modeling proton transfer is to treat the proton as a separate molecule, with its own potential parameters [46]. However, in order to produce reasonable results the charge assigned to the free proton has to be considerably lower than $+1 e$; for example Kathmann et al. used the value $+0.1627 e$. This counterintuitive adjustment decreases the chemical justification for the model, and raises the question of whether the dynamics predicted for the "partially charged protons" have any physical significance. However, as mentioned above, real proton transfer reactions are known to be influenced by quantum mechanical tunneling, which is replicated neither by molecular dynamics nor conventional quantum chemical methods, both of which assume atomic nuclei to move according to classical physics. Thus, a "partially charged" proton is not necessarily that much worse for modeling proton transfer than any other model based on classical nuclear motion.

\subsection{Investigating the fundamental chemistry of nucleating systems}

Another application of quantum chemical methods is the investigation of the fundamental chemical behaviour of molecular systems potentially relevant to nucleation. Within the field of tropospheric nucleation mechanisms, two questions which have merited considerable study under the last decade are the modeling of the hydration of sulfuric acid, and the role of ammonia in sulfuric acid-water nucleation.

\subsubsection{Sulfuric acid hydrates}

The concentration of water molecules in the atmosphere is around $10^{15} / \mathrm{cm}^{3}$, while that of sulfuric acid molecules rarely exceeds $10^{8} / \mathrm{cm}^{3}$. Thus, water molecules frequently collide with acid molecules. Chemical intuition and experiments $[48,49]$ show that sulfuric acid molecules gather water around them in the atmosphere: these small stable clusters containing sulfuric acid and water are called hydrates, and although stable, they have not nucleated. Hydrates correspond to a local formation free energy minimum, and to grow further they face an uphill of the 
TABLE 19.1 Gibbs free energy (at $298 \mathrm{~K}$ and $1 \mathrm{~atm}$ reference pressure) for the addition of water molecules to sulfuric acid, from various calculations and experiments. For the quantum chemical results, the use of harmonic or anharmonic vibrational frequencies has been indicated

\begin{tabular}{lll}
\hline & $\begin{array}{l}\mathrm{H}_{2} \mathrm{SO}_{4}+\mathrm{H}_{2} \mathrm{O} \Rightarrow \\
\mathrm{H}_{2} \mathrm{SO}_{4} \cdot \mathrm{H}_{2} \mathrm{O}\end{array}$ & $\begin{array}{l}\mathrm{H}_{2} \mathrm{SO}_{4} \cdot \mathrm{H}_{2} \mathrm{O}+\mathrm{H}_{2} \mathrm{O} \Rightarrow \\
\mathrm{H}_{2} \mathrm{SO}_{4} \cdot\left(\mathrm{H}_{2} \mathrm{O}\right)_{2}\end{array}$ \\
\hline B3LYP/6-311++G(2d,2p), harmonic [53] & $-0.6 \mathrm{kcal} / \mathrm{mol}$ & $-0.1 \mathrm{kcal} / \mathrm{mol}$ \\
B3LYP/D95++G(d,p), harmonic [44] & $-2.4 \mathrm{kcal} / \mathrm{mol}$ & $-2.0 \mathrm{kcal} / \mathrm{mol}$ \\
PW91/DNP, harmonic [54] & $-2.1 \mathrm{kcal} / \mathrm{mol}$ & $-1.4 \mathrm{kcal} / \mathrm{mol}$ \\
PW91/ATZ2P, harmonic [55] & $-2.6 \mathrm{kcal} / \mathrm{mol}$ & $-2.6 \mathrm{kcal} / \mathrm{mol}$ \\
Extrapolated MP4/ & $-3.4 \mathrm{kcal} / \mathrm{mol}$ & $-2.3 \mathrm{kcal} / \mathrm{mol}$ \\
aug-cc-pV(T + d)Z, anharmonic [38] & & \\
CNT [59] & $-4.1 \mathrm{kcal} / \mathrm{mol}$ & $-2.5 \mathrm{kcal} / \mathrm{mol}$ \\
Experimental [48] & $-3.6 \pm 1 \mathrm{kcal} / \mathrm{mol}$ & $-2.3 \pm 0.3 \mathrm{kcal} / \mathrm{mol}$ \\
\hline
\end{tabular}

free energy surface. Formation of the critical cluster from hydrates has a higher free energy barrier than formation from free sulfuric acid molecules, and taking into account the effect of hydrates lowers the nucleation rate [50,51]. Relevant hydrates contain only 1-5 water molecules and one sulfuric acid molecule, and thus they are small enough to be treated with quantum chemical methods. Several groups have studied the structure and energetics of different protolysis states of the small hydrates $[38,44,47,52-56]$. Although hydrates with two sulfuric acid molecules contain a negligible fraction of the total gas-phase sulfuric acid, they are important for the nucleation process, as the critical clusters (at least in pure sulfuric acid-water nucleation) probably contain more than one sulfuric acid molecule, and they have also been the focus of several studies [56-58].

Table 19.1 shows experimental results [48] and the results of Re et al. [44], Bandy and Ianni [53], Ding and Laasonen [54], Al Natsheh et al. [55] and Kurtén et al. [38] for the Gibbs free energies (at $298 \mathrm{~K}$ and a reference pressure of $1 \mathrm{~atm}$ for all reactants) for the first two water addition reactions to a sulfuric acid molecule. Figure 19.3 shows the corresponding relative concentrations of free sulfuric acid molecules, monohydrates and dihydrates at $298 \mathrm{~K}$ and $\mathrm{RH} 50 \%$. Only clusters with one sulfuric acid molecule are considered in this comparison. The prediction of CNT [59] (with the Clegg activity model [60]) is also included in the table and figure, and it matches the experiments rather well. The comparison between the results of Kurtén et al. (calculated using a computationally very expensive combination of MP2/aug-cc-pV(D + d)Z geometries and anharmonic vibrational frequencies, MP2/aug-cc-pV(T $+d) Z$ and MP4/aug-cc-pV $(\mathrm{D}+\mathrm{d}) \mathrm{Z}$ electronic energies), those of Re et al. (computed at the B3LYP/D95++(d,p) level), Bandy and Ianni (computed at the B3LYP/6-311++G(2d,2p level), Ding et al. (computed at the PW91/DNP level) and Al Natsheh et al. (computed at the PW91/ATZ2P level) show that both high-level electronic energies and advanced thermochemical approaches are needed before experimental results can be qualitatively replicated using systematically reliable theories. Heavily parameterized methods can give 


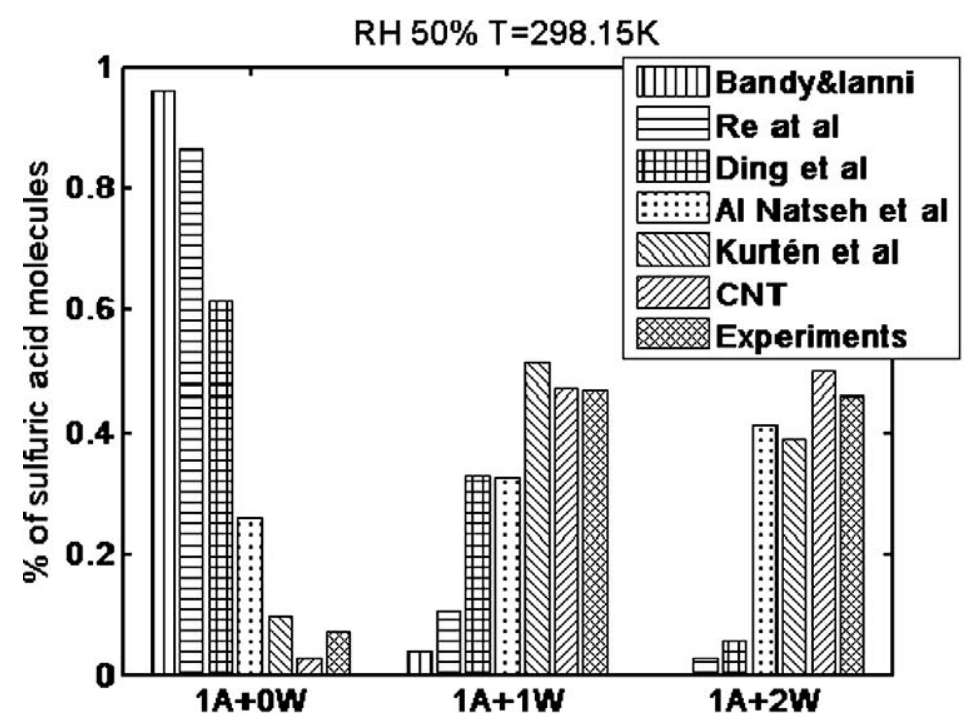

FIGURE 19.3 Distribution of sulfuric acid molecules to free acid molecules (1A $+0 \mathrm{~W})$ and clusters containing one sulfuric acid molecule and one water molecules (monohydrate, $1 \mathrm{~A}+1 \mathrm{~W}$ ) or two water molecule (dihydrate, $1 \mathrm{~A}+2 \mathrm{~W}$ ) according to experimental data [48], classical nucleation theory [59] and quantum chemical calculations of Re et al. [44], Bandy and Ianni [53], Ding and Laasonen [54], Al Natsheh et al. [55] and Kurtén et al. [38].

results compatible to experiments at the few existing experimental points by accident, but this is no guarantee that the results are realistic for other clusters.

\subsubsection{The role of ammonia in water-sulfuric acid nucleation}

Experimental studies have indicated that the presence of ammonia has a clear nucleation-enhancing effect [61] in the sulfuric acid-water system. However, different theoretical methods have yielded very different predictions for the strength of this effect. CNT predicts the enhancing effect to be stronger than what is experimentally observed. In terms of the average $\mathrm{NH}_{3}: \mathrm{H}_{2} \mathrm{SO}_{4}$ mole ratio of nucleating clusters in atmospheric conditions, state-of-the art thermodynamics and updated models based on classical nucleation theory $[60,62,63]$ have predicted ratios around or even above 1:1. In contrast, density functional studies on $\mathrm{H}_{2} \mathrm{SO}_{4}-\mathrm{H}_{2} \mathrm{O}-$ $\mathrm{NH}_{3}$ clusters containing one sulfuric acid molecule by Ianni and Bandy [64] at the B3LYP $/ 6-311++G(2 d, 2 p)$ level predicted that ammonia does not enhance particle formation, corresponding to a mole ratio of close to 0 . Larson et al. [65] studied the same cluster stoichiometries at the MP2/6-311++G(d,p) level, which yielded somewhat larger sulfuric acid-ammonia binding energies, and led them to conclude that ammonium bisulfate forms in the atmosphere. However, using their formation energetics (and computing free energies from their reported rotational constants and vibrational frequencies) together with atmospherically realistic temperatures and partial pressures for ammonia still leads to very low $\mathrm{NH}_{3}: \mathrm{H}_{2} \mathrm{SO}_{4}$ mole ratios for the one-acid clusters. 
An error analysis study by Kurtén et al. [66] found that the B3LYP density functional underestimates the binding energy of sulfuric acid-water and sulfuric acid-ammonia clusters compared to high-level coupled-cluster methods. The underestimation of B3LYP binding energies for sulfuric acid-water was also noted by Al Natsheh et al. [55] and Nadykto and Yu [67]. The neglect of vibrational anharmonicity also leads to underestimated binding energies. However, neither of these errors were large enough to explain the difference between experimental and computational predictions on the role of ammonia. More importantly, recent studies by Kurtén et al. [68], Nadykto and Yu [67] and Torpo et al. [69] showed that the main drawback of the earlier computational studies was not so much the quantum chemical method (though it plays a role, too) than the limitation of the cluster dataset to only one-acid clusters. Computations on larger clusters containing two or three sulfuric acid molecules demonstrate the nucleation-enhancing effect of ammonia. Specifically, the presence of ammonia at atmospherically realistic partial pressures was shown to significantly assist the growth of two-acid clusters to three-acid clusters [69]. This implies a lower limit of $1: 3$ for the $\mathrm{NH}_{3}: \mathrm{H}_{2} \mathrm{SO}_{4}$ mole ratio in atmospheric conditions, and qualitatively eliminates the contradiction between experimental and quantum chemical results.

In another recent study by Kurtén et al. [70], an upper limit to the $\mathrm{NH}_{3}: \mathrm{H}_{2} \mathrm{SO}_{4}$ mole ratio of atmospheric clusters was estimated by studying only the $\left(\mathrm{H}_{2} \mathrm{SO}_{4}\right)_{n}$. $\left(\mathrm{NH}_{3}\right)_{m}$ "core clusters" (with $n=2$ and $m=0, \ldots, 4$ ) without including any water molecules. This treatment was justified by the observation made in previous studies $[64,67,68]$ that the number of water molecules in the cluster affects the acid-ammonia binding relatively weakly, and also systematically: the addition of water molecules tends to increase the free energies of ammonia addition reactions (in other words make them less favorable thermodynamically). Thus, $\mathrm{NH}_{3}: \mathrm{H}_{2} \mathrm{SO}_{4}$ mole ratios computed for the "core clusters" represent an upper limit to the real mole ratios in atmospheric conditions. The omission of water molecules decreased the computational effort significantly, and the authors were able to use the quite high-level method RI-MP2/aug-cc-pV(T + d)Z/ / RI-MP2/aug-cc$\mathrm{pV}(\mathrm{D}+\mathrm{d}) \mathrm{Z}$. The effect of possible systematic errors due to, for example, basis-set effects, higher-level correlation or vibrational anharmonicity was assessed via a sensitivity analysis approach. The results indicate that $\mathrm{NH}_{3}: \mathrm{H}_{2} \mathrm{SO}_{4}$ mole ratios of atmospheric clusters are unlikely to exceed 1:1 in any atmospheric conditions, and are likely to be around 1:2 in most conditions. These mole ratios are somewhat lower that those typically measured for large $(>10 \mathrm{~nm}$ in diameter) clusters, which indicates that the composition of nucleating particles may differ significantly from larger particles. The precise reasons for the differences are still unknown, and worthy of further study.

\subsubsection{Studies on clusters containing other molecular species}

Quantum chemistry has also been applied to investigating whether other sulfurcontaining molecules than sulfuric acid itself might participate in nucleation reactions. Some recent experimental evidence $[71,72]$ indicates that the mixture of sulfuric acid and some intermediate products of the $\mathrm{SO}_{2}$ oxidation chain may promote nucleation even more effectively than sulfuric acid on its own. Similar 
speculations have also been presented in the past [73]. To our knowledge, no comprehensive and generally accepted explanation for the effect exists yet, though several studies have addressed issues related to the subject. For example, $\mathrm{SO}_{3} \cdot \mathrm{H}_{2} \mathrm{O}$ complexes have been studied by several groups, most recently by Morokuma and Muguruma [74], Meijer and Sprik [75], Larson et al. [76,77], Loerting and Liedl [42, 78], Ida et al. [79], Standard et al. [80] and Fliegl et al. [81]. (Note that most of these studies do not explicitly focus on atmospheric nucleation mechanisms.) One of the key results of these investigations has been that the presence of additional water molecules significantly lowers the activation barrier of the $\mathrm{SO}_{3}+\mathrm{H}_{2} \mathrm{O} \rightarrow \mathrm{H}_{2} \mathrm{SO}_{4}$ reaction. The studies also show that quantitatively accurate modeling of the formation and reactions of the $\mathrm{SO}_{3} \cdot \mathrm{H}_{2} \mathrm{O}$ complex require both high-level electronic energies (for example, the explicitly correlated RI-MP2-R12 calculations by Fliegl et al. [81] or the QCISD calculations by Standard et al. [80]) and, at least at lower temperatures, multidimensional tunneling corrections [42]. Especially the activation energies are sensitive to basis-set effects, as shown by Standard et al. [80].

The $\mathrm{HOSO}_{2}$ radical (formed in the addition of $\mathrm{OH}$ to $\mathrm{SO}_{2}$, the first step in the $\mathrm{SO}_{2}$ oxidation chain) and its complexes and reactions have also been the object of several studies, for example those by Majumdar et al. [82], Wierzejewska and Olbert-Majkut [83] and Aaltonen and Francisco [84]. Earlier studies [73] have speculated on the possible role of molecular species containing two sulfur atoms such as $\mathrm{H}_{2} \mathrm{~S}_{2} \mathrm{O}_{6}$ and $\mathrm{H}_{2} \mathrm{~S}_{2} \mathrm{O}_{8}$ in atmospheric nucleation processes. Such clusters have to our knowledge not been studied with quantum chemistry in the context of tropospheric nucleation, though Rosén et al. [85] used HF/6-31+G(d) calculations to qualitatively augment experimental results on the $\mathrm{H}_{2} \mathrm{~S}_{2} \mathrm{O}_{7}^{-} \cdot\left(\mathrm{H}_{2} \mathrm{SO}_{4}\right)_{x}$ ion cluster system, which might form from $\mathrm{HSO}_{4}^{-} \cdot\left(\mathrm{H}_{2} \mathrm{SO}_{4}\right)_{x}$ and $\mathrm{SO}_{3}$ in the stratosphere. Despite the numerous studies, there is yet no consensus on the mechanism of nucleation in the $\mathrm{SO}_{2}-\mathrm{H}_{2} \mathrm{O}-\mathrm{O}_{2}$-ultraviolet light system, and the topic will remain the focus of intensive studies. Quantum chemistry is likely to be a key tool in these investigations, as the relevant systems involve both radicals and chemical reactions, which are difficult to study using other methods such as classical potentials.

A few studies have also considered the effect of other inorganic species together with sulfuric acid or its precursors. Larson and Tao [77] and Pawlowski et al. [86] investigated $\mathrm{SO}_{3} \cdot \mathrm{NH}_{3} \cdot \mathrm{H}_{2} \mathrm{O}$ clusters, and concluded that in very high ammonia concentrations, $\mathrm{NH}_{3} \cdot \mathrm{SO}_{3}$ clusters rather than $\mathrm{H}_{2} \mathrm{SO}_{4}$-based clusters might act as nucleating agents. Ida et al. [79] also studied clusters containing $\mathrm{SO}_{3}$ along with both $\mathrm{H}_{2} \mathrm{O}, \mathrm{OH}, \mathrm{NH}_{3}$ and several other species (such as methylated ammonia), but did not speculate on the atmospheric implications of their results. Bienko and Latajka [87] studied $\mathrm{H}_{2} \mathrm{SO}_{4} \cdot \mathrm{CO}$ complexes, but focused mainly on their IR spectra, without much speculation of their potential role in the atmosphere.

DMS (dimethylsulfate) is produced by algae in the sea water, and is a likely nucleation precursor over the oceans. Its oxidation reactions yields $\mathrm{SO}_{2}$ and finally sulfuric acid. Other products of DMS oxidation than $\mathrm{SO}_{2}, \mathrm{SO}_{3}$ or $\mathrm{H}_{2} \mathrm{SO}_{4}$ have also been investigated using quantum chemistry, for example in the recent studies by Li et al. [88] and Wang [89], which both predicted that methanesulfonic acid (MSA), like sulfuric acid, is strongly hydrated in atmospheric conditions, and suggested that hydrated clusters of MSA might participate in nucleation processes. 
Some recent quantum chemical studies have addressed the participation of organic molecules in nucleation. Recent experimental evidence [90-92] indicates that various organic molecules, possibly together with sulfuric acid $[93,94]$ may be involved in some atmospheric nucleation events. There are innumerable quantum chemical studies of gas-phase organic reactions potentially relevant to aerosol processes, but few of these have focused specifically on nucleation. Exceptions among these are the PW91 study of Sloth et al. [95] on interaction energies between aerosol precursors formed in the photo-oxidation of $\alpha$-pinene, and the X3LYP study by Tong et al. [96] on secondary organic aerosol formation from reactions of aldehydes. Sulfuric acid-organic acid complexes have recently been investigated by Nadykto and Yu [67], who studied complexes of sulfuric acid with formic acid or acetic acid at the PW91/6-311++G(3df,3pd) level and by Zhang et al. [93], who considered complexes of sulfuric acid with aromatic acids formed in combustion processes, using the B3LYP functional with CCSD(T) energy corrections. Both of these studies found that sulfuric acid is quite strongly bound to organic acids, indicating that such clusters may play some role in nucleation. However, the experimental evidence [90-92,94] indicates that the sulfuric acid-organic nucleation mechanisms may involve real chemical reactions as opposed to simple clustering (and associated proton transfer reactions). An example of such a reaction was recently studied by Kurtén et al. [97], who investigated the reaction of sulfuric acid with stabilized Criegee Intermediates formed in the ozonolysis of biogenic terpenes using the B3LYP and RI-CC2 methods. The reaction was found to be essentially barrierless, but its atmospheric relevance depends strongly on the formation rates and lifetime of the biogenic stabilized Criegee Intermediates, for which no definite and reliable values are yet available.

Nucleation involving iodine oxide species has recently been investigated by several groups, some of which have supported their experimental work with quantum chemical computations. For example, Saunders and Plane [98] used a combination of electron microscopy and density functional theory (B3LYP with RRKM kinetics modeling) computations, and predicted that the ultra-fine iodine oxide particles formed in the coastal marine boundary layer consist of $\mathrm{I}_{2} \mathrm{O}_{5}$, while Begović et al. [99] computed energetics (using the G96PW91 functional) for HOIO formation reactions in order to explain observed (laboratory) mass spectra of iodine-containing aerosols.

Ion-induced nucleation has also been studied using quantum chemical methods. Low-level quantum chemistry calculations have been used to interpret and complement experimental results in studies by Froyd and Lovejoy [100] and Rosén et al. [85] on $\mathrm{HSO}_{4}^{-}$and $\mathrm{H}_{2} \mathrm{~S}_{2} \mathrm{O}_{7}^{-}$-based ion clusters, respectively. Recently, Nadykto et al. [101] showed that the sign preference in ion-induced nucleation (e.g. water tends to nucleate more efficiently on negatively charged ions) can be quite easily explained and predicted by quantum chemical calculations on small charged clusters. Kurtén et al. [38] recently studied the hydration of $\mathrm{HSO}_{4}^{-}$, and also tentatively concluded that the role of ammonia in ion-induced sulfuric acid-water nucleation is likely to be significantly smaller than in neutral sulfuric acid-water nucleation.

Obtaining qualitatively useful data for nucleation studies does not necessarily require the computation of quantitatively accurate free energies. For example, the 
relative importance of different nucleation pathways is primarily related to the differences in formation free energies between different types of clusters rather than to the absolute free energies. Even computational methods with relatively large systematic errors can thus yield useful and reliable data, as long as the errors are constant for the set of systems compared. For example, a test comparison [66] of 12 computational methods ( 3 theories and 4 basis sets, with and without counterpoise corrections) yielded differences as large as $5 \mathrm{kcal} / \mathrm{mol}$ for the absolute value of the binding energy of the $\mathrm{H}_{2} \mathrm{SO}_{4} \cdot \mathrm{NH}_{3}$ cluster, but all predicted values within about $2 \mathrm{kcal} / \mathrm{mol}$ from each other for the difference in binding energies of the $\mathrm{H}_{2} \mathrm{SO}_{4} \cdot \mathrm{NH}_{3}$ and $\mathrm{H}_{2} \mathrm{SO}_{4} \cdot \mathrm{H}_{2} \mathrm{O}$ clusters. The latter value, as discussed in [38] and [68] is much more important for assessing the role of ammonia in sulfuric acidwater nucleation. As another example, the studies of Froyd and Lovejoy [100] and Rosén et al. [85] show that even very modest HF-level calculations can provide qualitatively useful data that assists the interpretation of experimental results.

\subsection{Computing formation free energies for nucleating clusters}

The most ambitious application of quantum chemistry to nucleation is the calculation of the formation free energy of a critical cluster. In order to verify that a cluster is critical at the given conditions, the minimum requirement is that the free energy surface as a function of the molecular composition contains a maximum. Due to computational constraints, this has to our knowledge not been done for any system relevant to tropospheric nucleation mechanisms. For pure water clusters such data may exist, but as mentioned in Section 1, homogeneous nucleation of water does not occur anywhere in the atmosphere. Partial free energy surfaces restricted to the smallest cluster sizes have been computed by Kurdi and Kochanski [47], Re et al. [44], Arstila et al. [52], Ianni and Bandy [53,57], Al Natsheh et al. [55], Ding and Laasonen [54,58], Arrouvel et al. [56] and Kurtén et al. [38] for $\mathrm{H}_{2} \mathrm{SO}_{4}-\mathrm{H}_{2} \mathrm{O}$ clusters, by Ianni and Bandy [64], Larson et al. [65], Kurtén et al. [68,70], Nadykto and $\mathrm{Yu}$ [67] and Torpo et al. [69] for $\mathrm{H}_{2} \mathrm{SO}_{4}-\mathrm{NH}_{3}-\mathrm{H}_{2} \mathrm{O}$ clusters, and by Larson and Tao [77] and Pawlowski et al. [86] for $\mathrm{SO}_{3}-\mathrm{NH}_{3}-\mathrm{H}_{2} \mathrm{O}$ clusters.

For the calculations to be useful for computing formation free energies and further nucleation rates, both the electronic energy and the thermal contributions need to be computed using quite an advanced method to yield quantitatively reliable results. A recent high-level study [38] on $\mathrm{HSO}_{4}^{-} \cdot\left(\mathrm{H}_{2} \mathrm{O}\right)_{1, \ldots, 4}$ and $\mathrm{H}_{2} \mathrm{SO}_{4} \cdot\left(\mathrm{H}_{2} \mathrm{O}\right)_{1, \ldots, 4}$ clusters demonstrates that a combination of MP2/aug-cc$\mathrm{pV}(\mathrm{D}+\mathrm{d}) \mathrm{Z}$ geometries, high-level correlation (MP4) and large basis-set (aug-cc$\mathrm{pV}(\mathrm{T}+\mathrm{d}) \mathrm{Z})$ corrections, perturbative anharmonic vibrational frequency calculations and internal rotor analysis can replicate experimental formation enthalpies and entropies for very small clusters (see Figure 19.3). However, the computational effort involved is very large; the method could not be directly applied to clusters larger than $\mathrm{H}_{2} \mathrm{SO}_{4} \cdot\left(\mathrm{H}_{2} \mathrm{O}\right)_{2}$ or $\mathrm{HSO}_{4}^{-} \cdot\left(\mathrm{H}_{2} \mathrm{O}\right)_{2}$, and scaling-factor based extensions failed severely for the $\mathrm{HSO}_{4}^{-} \cdot\left(\mathrm{H}_{2} \mathrm{O}\right)_{4}$ cluster, presumably due to unidentified internal rotations.

In the future, the extent of accurate free energy surfaces will probably increase gradually. This is due both to the increases in computing power, and the 
development and testing of methods that allow a greater degree of accuracy for a certain amount of computing power. These methods include the resolution-ofidentity approximations, which have recently been used in atmospheric studies for example by Fliegl et al. [81] and Kurtén et al. [70], and the density fitting approximation, which has been successfully applied by Nadykto et al. [102] to the vibrational spectra of sulfuric acid monohydrate and formic acid dimer clusters. Intriguing possibilities are also offered by various QM/MM (Quantum Mechanics/Molecular Mechanics) methods and fragmentation or embedding models, see for example Heyden et al. [103] and Dahlke and Truhlar [104] for recent applications to water clusters, and Falsig et al. [105] for an atmospherically relevant study of phenol-water cluster reactions.

\section{Challenges}

One of the main difficulties of quantum chemical clusters studies is the generation of initial input structure for the geometry optimizations. The number of cluster conformers (structural isomers) increases combinatorially with the number of participating molecules, and a complete sampling of all conformers with quantum chemical methods becomes impossible already for relatively small clusters (around 6-8 molecules). Unfortunately, most cluster studies do not explicitly address the issue of input structure generation, which makes it difficult or impossible to reproduce their results, and decreases their usefulness as a starting point for further research. Presumably, they have usually employed a combination of chemical intuition and comparisons to earlier studies on similar systems. Classical potentials or semi-empirical methods together with various annealing simulations can be used to generate multiple conformers from a smaller number of initial structures, and lower-level quantum chemical computations are often used to narrow down the number of structures selected for the higher-level optimization. The development of generally available systematic Monte Carlo-based conformer sampling packages (for example within the Spartan 06 [106] program suite) will hopefully help decrease the randomness of the input structure generation process. In the future, we predict that input structures will increasingly be generated by a combination of system-specific interaction potentials with Monte Carlo-based sampling algorithms.

However, as the size of the studied cluster structures grows, it should be remembered that modeling the cluster as a single, harmonically vibrating and rigidly rotating minimum energy structure becomes an increasingly inadequate approach. In the context of atmospheric clusters, especially water molecules are quite weakly bound to the clusters, and the minimum energy geometry, harmonic oscillator and rigid rotor approximations are not well justified for extensively hydrated clusters. In a recent molecular mechanics study by Kathmann et al. [107], the effect of anharmonicity on the thermochemistry of hydrated ions was found to be notable for $i>4$ for $\mathrm{Na}^{+} \cdot\left(\mathrm{H}_{2} \mathrm{O}\right)_{i}$ clusters and $i>1$ for $\mathrm{Cl}^{-} \cdot\left(\mathrm{H}_{2} \mathrm{O}\right)_{i}$ clusters. It should be noted that Kathmann et al. use the term "anharmonicity" to cover 
two essentially separate issues: the anharmonicity of vibrational frequencies themselves, and the contribution of other conformers than the minimum energy (or minimum free energy) structure to the thermochemical parameters of the cluster. While the former can be accounted for by anharmonic vibrational calculations for smaller clusters, and various scaling factor approaches for larger clusters, the latter can not, according to Kathmann et al., be fully addressed by anything else than a complete sampling of the configuration space-an impossible task for even the computationally cheapest quantum chemical methods. As described in Section 3.3, we have recently [38] shown that a combination of anharmonic frequency calculations and internal rotation analysis can replicate the experimental thermochemical parameters for the very smallest sulfuric acid-water clusters. While anharmonic frequency calculations are computationally prohibitively expensive, internal rotor analysis is somewhat less demanding, and could potentially help decrease some of the worst errors induced by the harmonic approximation for mediumsized (on the order of 10 molecules) clusters. For qualitative studies, sensitivity analysis approaches such as that employed in our recent study [70] can also give order-of-magnitude estimates of the possible role of anharmonicity.

The apparent controversy between classical and quantum chemical modeling is in any case somewhat misleading. Real molecular clusters are both "quantum mechanical" and "anharmonic"; neither classical anharmonic nor quantum mechanical harmonic approaches can be expected to yield perfect results. The approaches should be seen as complementary rather than competing, with each method helping to complement the drawbacks of the other. For classical simulations, this could mean for example zero-point energy corrections (when they are not already implicitly included by fitting to experimental results), or correcting the computed potential energy surfaces using a limited set of quantum chemical single-point energy calculations. For quantum chemical simulations, it could imply more extensive conformational sampling using classical potentials, or QM/MM simulations with, for example, water molecules in outer hydration shells being treated using molecular dynamics. The ultimate objective would be to combine the chemical accuracy and universal nature of quantum chemistry with the extensive configurational sampling of Monte Carlo methods and the dynamic character of molecular dynamic studies.

\section{CONCLUSIONS}

Quantum chemical methods are valuable tools for studying atmospheric nucleation phenomena. Molecular geometries and binding energies computed using electronic structure methods can be used to determine potential parameters for classical molecular dynamic simulations, which in turn provide information on the dynamics and qualitative energetics of nucleation processes. Quantum chemistry calculations can also be used to obtain accurate and reliable information on the fundamental chemical and physical properties of molecular systems relevant to nucleation. Successful atmospheric applications include investigations on the hydration of sulfuric acid and the role of ammonia, sulfur trioxide and/or ions 
in sulfuric acid-water nucleation. Recently, atmospheric nucleation mechanisms involving short-lived precursors of $\mathrm{H}_{2} \mathrm{SO}_{4}$ or various organic molecules have also been investigated using quantum chemical methods. As these mechanisms often involve complicated chemical reactions, they can not reliably be studied using classical potentials, and future studies on these nucleation processes are likely to rely heavily on quantum chemical tools.

Unfortunately, quantitatively reliable quantum chemical calculations of nucleation rates for atmospherically relevant systems would require the application of both high-level electronic structure methods and complicated anharmonic thermochemical analysis to large cluster structures. Such computations are therefore computationally too expensive for currently available computer systems, and will likely remain so for the foreseeable future. Instead, a synthesis of different approaches will probably be necessary. In the future, successful nucleation studies are likely to contain combinations of the best features of both classical (Monte Carlo and molecular dynamics) and quantum chemical methods, with the ultimate objective being a chemically accurate, complete configurational sampling.

\section{ACKNOWLEDGEMENTS}

The authors thank the Academy of Finland for financial support, and Kai Nordlund, Ismo Napari, Kari E. Laasonen, Adam Foster and Martta Salonen for assistance.

\section{REFERENCES}

[1] D.V. Spracklen, K.S. Carslaw, M. Kulmala, V.-M. Kerminen, G.W. Mann, S.-L. Sihto, Atmos. Chem. Phys. 6 (2006) 5631.

[2] The full report will be published by Cambridge Univ. Press, New York. Online version available at: http://ipcc-wg1.ucar.edu/wg1/wg1-report.html.

[3] S. Von Klot, A. Peters, P. Aalto, T. Bellander, N. Berglind, D. D’Ippoliti, R. Elosua, A. Hörmann, M. Kulmala, T. Lanki, H. Löwel, J. Pekkanen, S. Picciotto, J. Sunyer, F. Forastriere, the \{HEAPSS\} study group, Circulation 112 (2005) 3073.

[4] M. Kulmala, H. Vehkamäki, T. Petäjä, M. Dal Maso, A. Lauri, V.-M. Kerminen, W. Birmili, P.H. McMurry, J. Aerosol Sci. 35 (2004) 143.

[5] C.D. O’Dowd, J.L. Jimenez, R. Bahreini, R.C. Flagan, J.H. Seinfeld, K. Hämeri, L. Pirjola, M. Kulmala, S.G. Jennings, T. Hoffmann, Nature 417 (2002) 632.

[6] M. Vana, E. Tamm, U. Horrak, A. Mirme, H. Tammet, L. Laakso, P.P. Aalto, M. Kulmala, Atmos. Res. 82 (2006) 536.

[7] F.L. Eisele, E.R. Lovejoy, E. Kosciuch, K.F. Moore, R.L. Mauldin III, J.N. Smith, P.H. McMurry, K. Iida, J. Geophys. Res. (D) 111 (2006) D04305.

[8] V. Kanawade, S.N. Tripathi, J. Geophys. Res. (D) 111 (2006) D02209.

[9] J.E. McDonald, Am. J. Phys. 31 (1963) 31.

[10] F.F. Abraham, Homogeneous Nucleation Theory, Academic Press, New York, 1974.

[11] D.D. Kashchiev, Nucleation-Basic Theory with Applications, Butterworth-Heinemann, Oxford, 2000.

[12] H. Reiss, J. Chem. Phys. 18 (1950) 840.

[13] D. Stauffer, J. Aerosol Sci. 7 (1976) 319.

[14] J.B. Zeldovich, Acta Physicochim. U.R.S.S. XVIII (1943) 1/22/01. 
[15] J.B. Zeldovich, Zh. Eksp. Theor. Fiz. 12 (1942) 525.

[16] H. Vehkamäki, Classical Nucleation Theory in Multicomponent Systems, Springer, BerlinHeidelberg, 2006.

[17] J. Merikanto, E. Zapadinsky, A. Lauri, H. Vehkamäki, Phys. Rev. Lett. 98 (2007) 145702.

[18] R. Evans, Adv. Phys. 28 (1979) 143.

[19] D. Henderson, Fundamentals of Inhomogeneous Fluids, Marcel Dekker, New York, 1992.

[20] A. Laaksonen, I. Napari, J. Phys. Chem. B 105 (2001) 11678.

[21] K. Binder, J. Horbach, W. Kob, W. Paul, F. Varnik, J. Phys. Condens. Matter 16 (2004) S429.

[22] N. Metropolis, A.W. Rosenbluth, M.N. Rosenbluth, A.H. Teller, E. Teller, J. Chem. Phys. 21 (1953) 1087.

[23] J. Yao, R.A. Greenkorn, C. Chao, Mol. Phys. 46 (1982) 587.

[24] J. Merikanto, H. Vehkamäki, E. Zapadinsky, J. Chem. Phys. 121 (2004) 914.

[25] I. Napari, H. Vehkamäki, J. Chem. Phys. 125 (2006) 094313.

[26] S.A. Harris, I.J. Ford, J. Chem. Phys. 118 (2003) 9216.

[27] E. Kelly, M. Seth, T. Ziegler, J. Phys. Chem A 108 (2004) 2167.

[28] D.C. Rapaport, The Art of Molecular Dynamics Simulation, second ed., Cambridge University Press, Cambridge, UK, 2004.

[29] F. Jensen, Introduction to Computational Chemistry, second ed., Wiley, West Sussex, UK, 2007.

[30] S. Tsusuki, H.P. Lüthi, J. Chem. Phys. 114 (2001) 3949.

[31] T. van Mourik, R.J. Gdanitz, J. Chem. Phys. 116 (2002) 9620.

[32] Y. Zhao, N.E. Schultz, D.G. Truhlar, J. Chem. Theory Comput. 2 (2006) 364.

[33] Y.-K. Choe, E. Tsuchida, T. Ikeshoji, J. Chem. Phys. 126 (2007) 154510.

[34] G.M. Chaban, J.O. Jung, R.B. Gerber, J. Phys. Chem. A 104 (2000) 2772.

[35] L. Pele, B. Brauer, R.B. Gerber, Theor. Chem. Acc. 117 (2007) 69.

[36] V. Barone, J. Chem. Phys. 122 (2005) 014108.

[37] Y. Miller, G.M. Chaban, R.B. Gerber, J. Phys. Chem. A 109 (2005) 6565.

[38] T. Kurtén, M. Noppel, H. Vehkamäki, M. Salonen, M. Kulmala, Boreal Environ. Res. 12 (2007) 431.

[39] D.W. Brenner, Phys. Rev. B 42 (1990) 9458.

[40] A.C.T. van Duin, S. Dasgupta, F. Lorant, W.A. Goddard III, J. Phys. Chem. A 105 (2001) 9396.

[41] R.J. McMahon, Science 299 (2003) 833.

[42] T. Loerting, K.R. Liedl, J. Phys. Chem. A 105 (2001) 5137.

[43] C.-G. Ding, T. Taskila, K. Laasonen, A. Laaksonen, Chem. Phys. 287 (2003) 7.

[44] S. Re, Y. Osamura, K. Morokuma, J. Phys. Chem. A 103 (1999) 3535.

[45] I. Kusaka, Z.-G. Wang, J. Seinfeld, J. Chem. Phys. 108 (1998) 6829.

[46] S.M. Kathmann, B.N. Hale, J. Phys. Chem. B 105 (2001) 11719.

[47] L. Kurdi, E. Kochanski, Chem. Phys. Lett. 158 (1989) 111.

[48] D.R. Hanson, F. Eisele, J. Phys. Chem. A 104 (2000) 1715.

[49] J.J. Marti, A. Jefferson, X.P. Cai, C. Richert, P.H. McMurry, F. Eisele, J. Geophys. Res. 102 (1997) 3725.

[50] R.H. Heist, H. Reiss, J. Chem. Phys. 61 (1974) 573.

[51] A. Jaecker-Voirol, P. Mirabel, H. Reiss, J. Chem. Phys. 87 (1987) 4849.

[52] H. Arstila, K. Laasonen, A. Laaksonen, J. Chem. Phys. 108 (1998) 1031.

[53] A.R. Bandy, J.C. Ianni, J. Phys. Chem. A 102 (1998) 6533.

[54] C.-G. Ding, K. Laasonen, Chem. Phys. Lett. 390 (2004) 307.

[55] A. Al Natsheh, A.B. Nadykto, K.V. Mikkelsen, F. Yu, J. Ruuskanen, J. Phys. Chem. A 108 (2004) 8914;

Correction published in J. Phys. Chem. A 110 (2006) 7982.

[56] C. Arrouvel, V. Viossat, C. Minot, J. Mol. Struct. THEOCHEM 718 (2005) 71.

[57] J.C. Ianni, A.R. Bandy, J. Mol. Struct. THEOCHEM 497 (2000) 19.

[58] C.-G. Ding, K. Laasonen, A. Laaksonen, J. Phys. Chem. A 107 (2003) 8648.

[59] M. Noppel, H. Vehkamäki, M. Kulmala, J. Chem. Phys. 116 (2002) 218.

[60] S.L. Clegg, P. Brimblecombe, A.S. Wexler, J. Phys. Chem. A 102 (1998) 2137.

[61] S.M. Ball, D.R. Hanson, F.L. Eisele, P.H. McMurry, J. Geophys. Res. D 104 (1999) 23709.

[62] H. Vehkamäki, I. Napari, M. Kulmala, M. Noppel, Phys. Rev. Lett. 93 (2004) 148501.

[63] T. Anttila, H. Vehkamäki, I. Napari, M. Kulmala, Boreal Environ. Res. 10 (2005) 511. 
[64] J.C. Ianni, A.R. Bandy, J. Phys. Chem. A 103 (1999) 2801.

[65] L.J. Larson, A. Largent, F.-M. Tao, J. Phys. Chem. A 103 (1999) 6786.

[66] T. Kurtén, M.R. Sundberg, H. Vehkamäki, M. Noppel, J. Blomqvist, M. Kulmala, J. Phys. Chem. A 110 (2006) 7178.

[67] A.B. Nadykto, F. Yu, Chem. Phys. Lett. 435 (2007) 14.

[68] T. Kurtén, L. Torpo, C.-G. Ding, H. Vehkamäki, M.R. Sundberg, K. Laasonen, M. Kulmala, J. Geophys. Res. 112 (2007) D04210.

[69] L. Torpo, T. Kurtén, H. Vehkamäki, M.R. Sundberg, K. Laasonen, M. Kulmala, J. Am. Chem. Soc. A 111 (2007) 10671.

[70] T. Kurtén, L. Torpo, M.R. Sundberg, V.-M. Kerminen, H. Vehkamäki, M. Kulmala, Atmos. Chem. Phys. Discuss. 7 (2007) 2937.

[71] T. Berndt, O. Böge, F. Stratmann, J. Heintzenberg, M. Kulmala, Science 307 (2005) 698.

[72] T. Berndt, O. Böge, F. Stratmann, Geophys. Res. Lett. 33 (2006) L15817.

[73] J.P. Friend, R.A. Burnes, R.M. Vasta, J. Phys. Chem. 84 (1980) 2423.

[74] K. Morokuma, C. Muguruma, J. Am. Chem. Soc. 116 (1994) 10316.

[75] E.J. Meijer, M. Sprik, J. Phys. Chem. A 102 (1998) 2893.

[76] L.J. Larson, M. Kuno, F.-M. Tao, J. Chem. Phys. 112 (2000) 8830.

[77] L.J. Larson, F.-M. Tao, J. Phys. Chem. A 105 (2001) 4344.

[78] T. Loerting, K.R. Liedl, Proc. Natl. Acad. Sci. 97 (2000) 8874.

[79] B.N. Ida, P.S. Fudacz, D.H. Pulsifer, J.M. Standard, J. Phys. Chem. A 110 (2006) 5831.

[80] J.M. Standard, I.S. Buckner, D.H. Pulsifer, J. Mol. Struct. THEOCHEM 673 (2004) 1.

[81] H. Fliegl, A. Glöß, O. Welz, M. Olzmanna, W. Klopper, J. Chem. Phys. 125 (2006) 054312.

[82] D. Majumdar, G.-S. Kim, J. Kim, K.S. Oh, J.Y. Lee, K.S. Kima, W.Y. Choi, S.-H. Lee, M.-H. Kang, B.J. Mhin, J. Chem. Phys. 112 (2000) 723.

[83] M. Wierzejewska, A. Olbert-Majkut, J. Phys. Chem. A 111 (2007) 2790.

[84] E.T. Aaltonen, J.S. Francisco, J. Phys. Chem. A 107 (2003) 1216.

[85] S. Rosén, K.D. Froyd, J. Curtius, E.R. Lovejoy, Int. J. Mass Spectrom. 232 (2004) 9.

[86] P.M. Pawlowski, S.R. Okimoto, F.-M. Tao, J. Phys. Chem. A 107 (2003) 5327.

[87] A.J. Bienko, Z. Latajka, Chem. Phys. 282 (2002) 207.

[88] S. Li, W. Qian, F.-M. Tao, J. Phys. Chem. A 111 (2007) 190.

[89] L. Wang, J. Phys. Chem. A 111 (2007) 3642.

[90] B. Bonn, G. Schuster, G.K. Moortgat, J. Phys. Chem. A 106 (2002) 2869.

[91] B. Bonn, G.K. Moortgat, Geophys. Res. Lett. 30 (2003) 1585.

[92] N.L. Ng, J.H. Kroll, M.D. Keywood, R. Bahreini, V. Varutbangkul, R.C. Flagan, J.H. Seinfeld, A. Lee, A.H. Goldstein, Environ. Sci. Technol. 40 (2006) 2283-2297.

[93] R. Zhang, I. Suh, J. Zhao, D. Zhang, E.C. Fortner, X. Tie, L.T. Molina, M.J. Molina, Science 2004 (2004) 1487.

[94] J.D. Surratt, J.H. Kroll, T.E. Kleindienst, E.O. Edney, M. Claeys, A. Sorooshian, N.L. Ng, J.H. Offenberg, M. Lewandowski, M. Jaoui, R.C. Flagan, J.H. Seinfeld, Environ. Sci. Technol. 41 (2007) 517.

[95] M. Sloth, M. Bilde, K.V. Mikkelsen, Mol. Phys. 102 (2004) 2361.

[96] C. Tong, M. Blanco, W.A. Goddard III, J.H. Seinfeld, Environ. Sci. Technol. 40 (2006) 2333.

[97] T. Kurtén, B. Bonn, H. Vehkamäki, M. Kulmala, J. Phys. Chem. A 111 (2007) 3391.

[98] R.W. Saunders, J.M.C. Plane, Environ. Chem. 2 (2005) 299.

[99] N. Begović, Z. Marković, S. Anić, L. Kolar-Anić, Environ. Chem. Lett. 2 (2004) 65.

[100] K.D. Froyd, E.R. Lovejoy, J. Phys. Chem. A 107 (2003) 9812.

[101] A.B. Nadykto, A. Al Natsheh, F. Yu, K.V. Mikkelsen, J. Ruuskanen, Phys. Rev. Lett. 96 (2006) 125701.

[102] A.B. Nadykto, H. Du, F. Yu, Vib. Spectrosc. 44 (2007) 286.

[103] A. Heyden, H. Lin, D.G. Truhlar, J. Phys. Chem. B 111 (2007) 2231.

[104] E.E. Dahlke, D.G. Truhlar, J. Chem. Theory Comput. 3 (2007) 46.

[105] H. Falsig, A. Gross, J. Kongsted, A. Osted, M. Sloth, K.V. Mikkelsen, O. Christiansen, J. Phys. Chem. A 110 (2006) 660.

[106] Wavefunction, Inc., Spartan ‘06, Wavefunction, Inc. Irvine, CA (2006). See also: www.wavefun. com.

[107] S. Kathmann, G. Schenter, B. Garrett, J. Phys. Chem. C 111 (2007) 4977. 